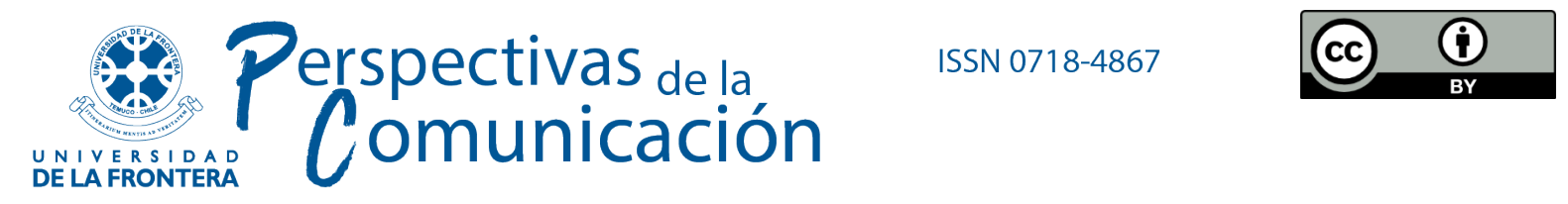

Artículo

\title{
NUEVOS ESCENARIOS EDUCATIVOS A PARTIR DEL COVID-19 EN LA EDUCACIÓN UNIVERSITARIA
}

DOI: POR DEFINIR

Dra. Almudena Barrientos-Báez

Universidad Complutense de Madrid, España

almbarri@ucm.es

ORCID iD: https://orcid.org/oooo-0001-9913-3353

Mg. Loreto González-Suazo

Universidad de Valparaíso, Chile

loreto.gonzalez@uv.cl

ORCID iD: https://orcid.org/oooo-0oo1-7116-6882

Dr. David Caldevilla-Domínguez

Universidad Complutense de Madrid, España

davidcaldevilla@ccinf.ucm.es

ORCID iD: https://orcid.org/o0oo-0002-9850-1350

Recibido el 2021-02-08

Revisado el 2021-10-26

Aceptado el 2021-10-26

Publicado el 2021-12-15

\section{Resumen}

El presente artículo reflexiona en torno a los nuevos escenarios surgidos en la Educación Superior, a partir de las necesidades planteadas por la COVID-19 y en función del contexto donde han sido implantados. En específico abordaremos las prácticas educativas que se orientan hacia el uso de las tecnologías de la información y comunicación (TIC), en el contexto de la pandemia COVID-19 desde una perspectiva crítica, para así visibilizar las tensiones y lógicas educativas que se están transformando, sus logros y límites.

Palabras clave: Comunicación, educación, COVID-19, currículo, TIC, universidad. 


\title{
NEW EDUCATIVE SCENARIOS AFTER COVID-19 IN HIGHER EDUCATION
}

\begin{abstract}
This article reflects on the new scenarios that have arisen in Higher Education, based on the needs raised by COVID-19 and depending on the context where they have been implemented. Specifically, we will address the educational practices that are oriented towards the use of information and communication technologies (ICT), in the context of the COVID-19 pandemic from a critical perspective, to make visible the tensions and educational logics that are being transformed, its achievements and limits.
\end{abstract}

Keywords: Communication, education, COVID-19, CV, ICT, university. 


\section{Introducción}

El presente artículo tiene como propósito analizar el área de las tecnologías de la información y la comunicación (TIC) en la educación superior en el contexto del COVID-19, que está experimentando la educación superior. Este desafío requiere esfuerzos desde un horizonte que permita abordar temáticas educativas que están en las fronteras de nuestra educación y que necesitan de una especial atención y cuidado. Este nuevo paradigma supone la obligación de comprender el 'nuevo' significado de educar y ha de llevar a cuestionar las lógicas educativas tradicionales de la educación superior, e incluso preguntarse si las tecnologías de la información y la comunicación pueden contribuir a este cambio de modelo que ha sido acelerado, pues ya existía, por la pandemia COVID-19 que vive el mundo.

Estos cuestionamientos han llevado a afrontar problemáticas relativas al currículum, en especial a la derivada de la posibilidad de que en la educación superior se pueda construir un corpus que se oriente a la reflexión sobre la práctica comunicativo-educativa, la cual exprese la acción como expresión de su valor; esto supone según Sacristán (1991): “que es en la práctica donde todo proyecto, toda idea, toda intención, se hace realidad de una forma $\mathrm{u}$ otra; se manifiesta, adquiere significación y valor, independientemente de las declaraciones y propósitos de partida” (p. 4). Además, estas problemáticas ponen la atención en el cuestionamiento de las prácticas educativas con las que se lleva a cabo un proyecto de enseñanza, planteando las siguientes interrogantes en la doble variante comunicativa y docente: ¿cómo las prácticas educativas que se orientan hacia el uso educativo de las TIC en la educación superior pueden tensionar al sistema educativo formal?, ¿de qué manera las prácticas educativas que se orientan hacia el uso educativo de las TIC pueden contribuir a la transformación de la educación superior? Hay una extensa literatura reciente de primer nivel al respecto que ayuda a entender la importancia que la Academia está dando a estas posibilidades visible, por ejemplo, en Monzalvo et al. (2018), en Hernández-Quintana (2019), en Celaya et al. (2020) e incluso proyectos nacionales e internacionales de primer nivel y en variados ámbitos del saber, como los propuestos por Colás-Bravo et al. (2019), Botella-Nicolás et al. (2019), Moreira et al. (2019) o el transdisciplinar García-Marín (2020). Llegados a este punto, es básico generar programas de control para que esta tendencia a la digitalización sea eficaz (Mears-Delgado y Marzal, 2018). Son arquetípicas las palabras de Blanco (2018), que sirven de toda una declaración de intenciones: "Las tecnologías de la información y la comunicación (TIC) aspiran a ser una pieza clave en el impulso, transferencia y gestión de conocimientos para fortalecimiento de los actores de la cooperación descentralizada para el desarrollo humano local”.

Perspectivas de la Comunicación - Vol. 14 - No 2 - 2021 - pp. 149-170

Universidad de la Frontera - Chile 
La aportación de este artículo es mostrar las diversas tensiones, preguntas y desafíos que implica apostar por la construcción de una nueva educación para una nueva sociedad en línea con trabajos como Salas-Rueda y Vázquez-Estupiñán (2017); Novoa (2019); MascarellPaláu (2019; 2020); Salas-Rueda y Salas-Rueda (2019); Salas-Rueda y Salas-Silis (2019); Gil-Villa et al. (2020); Pontes et al. (2020); Tabernero et al. (2020); Fernández-Ruiz (2020); Ortiz et al. (2019) y Valbuena-Bohórquez y Alvarado-Ortiz (2020) sólo por citar algunos de los más recientes entre los señeros.

\section{Método}

Dirigido a analizar en profundidad los nuevos escenarios educativos a partir del COVID-19 en la Educación Superior. En específico, nos aproximaremos a abordar las prácticas educativas que se orientan hacia el uso de las TIC en el contexto de la pandemia COVID-19. El método empleado de estudio será deductivo ya que partiremos de una premisa general y a partir de ahí podremos obtener conclusiones con la observación y análisis de las diferentes investigaciones y estudios realizados hasta el momento. Se ha realizado un análisis de tipo descriptivo, de recopilación y reelaboración de fuentes. Además, se ha llevado a cabo un relevamiento bibliográfico para poder identificar los trabajos referidos a la enseñanza universitaria y la pandemia del COVID-19.

\section{Resultados}

\subsection{El Contexto: Las TIC en el Campo Educativo}

En los últimos años del siglo XX, las TIC han tenido una expansión sorprendente en todos los ámbitos del quehacer humano (Maniglio, 2016), convirtiéndose en herramientas fundamentales para el desarrollo y cambio de la sociedad, por lo tanto, en el marco de una sociedad globalizada los sistemas educativos han tenido que adoptar e instalar las TIC en sus instituciones educativas, de tal forma que los ciudadanos del siglo XXI logren desenvolverse con éxito en la sociedad del conocimiento y, más que eso, se transformen no sólo en consumidores de información, sino en productores de conocimiento. Las AVA -Aula Virtual de Aprendizaje- y los MOOC - Massive Open Online Course o Cursos en línea masivos abiertos-han supuesto en todos los sentidos un avance y aclimatación a las nuevas TIC para los sistemas educativos, por lo que actualmente todos los cursos oficiales y privados suelen ofertar ese tipo de opciones, ya de apoyo, ya únicas (Rajas et al., 2018) y más desde la pandemia debido a sus problemas de movilidad. 
Ante esto, se hizo necesario acercar las TIC a los ciudadanos implementando proyectos educativos que estuvieran en directa relación con el quehacer de los ciudadanos del siglo XXI. En este sentido hay programas en todos los países y en todos los niveles educativos, como es el caso de Brasil en educación superior (Tavares, 2019) o el de Chile, quien "bajo el marco de acción de los programas de mejoramiento de la calidad y equidad de la reforma educacional, que se implementaron a partir de 1990, surge el Proyecto Enlaces, el cual buscó desde sus inicios instalar una infraestructura informática que uniese a personas, proyectos, experiencias educativas y que redujera el aislamiento de muchas escuelas e instituciones educativas, a través de una red educativa nacional, de tal forma que, gradualmente, el sistema educativo nacional iniciara un proceso de integración curricular de las TIC en las escuelas y liceos de Chile" (Vidal et al., 2009).

Las TIC y su vertiginosa incorporación al campo educativo, requiere que no sólo se quede en un nivel de infraestructura y equipamiento tecnológico, sino que además se pueda avanzar hacia un uso educativo como una herramienta, donde se pueda apostar por una integración crítica, en la cual se defina el qué, por qué y para qué de su incorporación y aprovechamiento. Hay que ver a las tecnologías como medio y recurso didáctico, mas no como la panacea que resolverá las problemáticas dentro del campo educativo. Esta afirmación lleva a no sobredimensionarlas y establecer orientaciones para su uso, logrando así soluciones pedagógicas y no tecnológicas. Esto se refiere a que "la incorporación de las TIC como mediadoras del proceso de aprendizaje nos lleva a valorar y a reflexionar respecto a la eficacia de la enseñanza" (Fandos et al., 2002). Para lograr el uso crítico de las tecnologías, y poder reconfigurar estos nuevos escenarios educativos, es necesaria la formación y el perfeccionamiento constante, donde las tecnologías sean un medio facilitador de los procesos de enseñanza-aprendizaje, el cual permita generar metodologías diversas, transformando las estructuras organizativas, generando dinámicas de motivación, e impulsando sobre todo, el cambio hacia un uso crítico, didáctico y pedagógico de las TIC. Esta situación es incluso más relevante ahora por el contexto mundial relacionado con la experiencia de estar en un proceso de pandemia, el cual nos va mostrando día a día que la formación de los estudiantes en la educación superior no sólo implica comprender y utilizar las TIC como mediadoras del aprendizaje, sino que, con mayor fuerza, se instalan como la posibilidad de aprender remotamente y generar un conocimiento en red.

Este tipo de enseñanza ha calado incluso en sectores de la población ajenos al sistema educativo reglado tradicional, como la tercera edad, ya que en opinión de Barrientos-Báez

Perspectivas de la Comunicación - Vol. 14 - No 2 - 2021 - pp. 149-170

Universidad de la Frontera - Chile 
et al. (2017) estas tecnologías ayudan a la integración social de dicho colectivo, a eliminar la brecha digital y a mejorar su nivel intelectual y detener el deterioro cognitivo, algo muy importante para las Administraciones.

\subsection{Tecnologías de la información y comunicación como herramientas para el aprendizaje}

Como se ha venido señalando, las TIC se constituyen como una herramienta pertinente para el aprendizaje en la sociedad del conocimiento en la que nos encontramos, sin embargo, el sistema educativo se halla ante un reto de importantes dimensiones: cuestionarse a sí mismo para repensar sus objetivos, metodologías y organización. Estos cuestionamientos se han visto en jaque a partir de la crisis sanitaria mundial, la cual no solo ha puesto al sistema educativo en la coyuntura de pasar de la docencia presencial a la virtual, sino que ha mostrado la incapacidad de muchos segmentos respecto de cómo abordar la educación superior en el entendido de comprender que las diferentes comunidades universitarias tienen diversas características y complejidades propias. En este sentido, las instituciones de educación universitaria son presentadas en dos escenarios distintos: adaptarse tecnológicamente a los cambios educativos que ha provocado la pandemia del COVID-19 (adaptación del currículo y perfiles de egreso en contexto de flexibilidad curricular), y por otro lado, a comenzar a instalar y comprender que el uso educativo de las TIC ya no es lejano sino que es aquí y ahora, lo que demanda una mayor capacitación por parte de académicos y académicas que transitan y transforman sus prácticas educativas a nuevas formas de enseñar. Las dinámicas propias que se daban dentro del aula han cambiado, y ahora el espacio/tiempo en donde se desarrolló la relación de enseñanza y aprendizaje está mediado por plataformas digitales, lo que nos va mostrando otros tipos de roles y relaciones entre docentes y estudiantes, lo que va generando nuevos tipos de conocimientos y contenidos.

Es por ello por lo que más allá de que las TIC sean una herramienta, es necesario que se generen las condiciones administrativas y organizativas dentro del sistema educativo, para que la incorporación de las TIC sea efectiva. Una de las consecuencias de esta nueva "no normalidad" es que las instituciones de educación superior gestionan nuevas líneas de funcionamiento y desarrollo académico dentro del aula. Necesitamos como institución educativa acercarnos a una concepción de la educación que vaya más allá de la rutina y la memorización de contenidos "en definitiva, un nuevo modo de hacer las cosas que lleven a repensar el modo en que se produce el proceso de enseñanza-aprendizaje” (CasanovaCorrea, 2007, p. 115). 
La reinterpretación del concepto de educación requiere detectar nuevas necesidades para el desarrollo de la enseñanza y el aprendizaje. Esto en la práctica no es tan sencillo de llevar a cabo, ya que requiere un cambio de actitudes de toda la comunidad educativa. El COVID-19 nos ha presionado a pensar en modelos educativos más flexibles y abiertos, donde el docente se convierta en el diseñador y orientador de espacios educativos novedosos para que los procesos de enseñanza - aprendizaje de los estudiantes tengan nuevas dimensiones, y el mismo estudiante pueda construir sus conocimientos. En este sentido, el estudiante debe tener un alto grado de motivación y de autonomía en el estudio, así como la capacidad para auto organizar su tiempo de forma compatible con todas sus otras ocupaciones personales y profesionales. La Inteligencia Emocional enseñada dentro de un modelo transversal de educación sería una herramienta fundamental para elaborar el cambio necesario en la formación de docentes y discentes. Según Barrientos-Báez et al. (2019) ésta radica en la capacidad de controlar y gestionar positivamente las emociones propias y ajenas, en un escenario cualquiera, donde se producen experiencias y cambios como parte del proceso de aprendizaje personal. Ahora bien, esta situación que nos presiona a partir del COVID-19 también nos abre otras aristas de la relación educativa, en el sentido de que si bien las instituciones de educación superior están adaptándose a estos nuevos escenarios, no es menos ciertos que los estudiantes también están sufriendo procesos de cambios profundos en la cosmovisión de su aprendizaje, un ejemplo de ello es darse cuenta que ahora los discentes, si bien manejan las redes sociales y se podría decir que son "nativos digitales", esta natividad o expertos en el dominio de las TIC, no está muy cerca en lo que respecta al uso educativo de las mismas, es decir, para los y las estudiantes es imprescindible educarse mediante tecnologías de la educación y comunicación y dejar de lado la dimensión de diversión con la que ellos principalmente la utilizan, lo que nos hace inferir que el aprendizaje que están teniendo quizás no alcanza a resolver todas las inquietudes del estudiante y puede comenzar a producir estados de ansiedad entre otros. Lo más destacable es la necesidad de generar un nuevo ambiente de aprendizaje (Gallego-Torres, 2020). Como apunta Barrientos-Báez (2016), se cuenta hoy en día con unas herramientas que permiten que los procesos de aprendizaje sean mucho más personalizados, mucho más flexibles. En este caso concreto, la formación en Inteligencia Emocional y TIC como elementos transversales es clave para el correcto desempeño profesional futuro. 


\subsection{Nuevo Papel del Docente en el Contexto COVID-19}

La incorporación de las TIC no sólo impacta en nuestra diaria cotidianeidad, sino en la forma de relacionarnos unos con otros (Caldevilla-Domínguez et al., 2019; 2020). En este contexto que ya es de común conocimiento vamos presenciando que las relaciones sociales que se establecen por ejemplo dentro del aula como escenario social, ya son de otro tipo, donde se ha transitado de una enseñanza centrada en el profesor a una enseñanza centrada en el estudiante y sus necesidades de conocimiento. Esto significa que debemos lograr identificar como institución educativa las nuevas necesidades de los estudiantes, para que puedan adquirir los conocimientos y habilidades esenciales encaminadas a una adaptación continua a los cambios a veces no previsibles del siglo XXI.

Es así como nos encontramos en un profundo tránsito desde la lógica presencial a una lógica virtual interactiva y motivadora que requiere cambios en los docentes, en los estudiantes, y en la comunidad educativa en su conjunto, para que las TIC puedan ser incorporadas. Es necesario que se cumplan ciertos principios básicos que tengan relación con el cambio de perspectiva y papeles que tanto el profesor como el estudiante deben establecer en los contextos educativos, como también en la necesidad de integrar las tecnologías a todo programa de formación docente. Esto implica según Quiroz (2012) que: "A lo largo de toda su experiencia educativa, los futuros docentes deben aprender de forma práctica acerca del uso de la tecnología y de las formas en que ésta puede incorporarse a sus clases”. Lo que plantea el autor requiere no limitar las experiencias relacionadas con las tecnologías a un único curso o a una única metodología para que los docentes interactúen con ellas, sino que los docentes durante su formación deberían controlar una amplia gama de tecnologías educativas, que partan de las experiencias introductorias con el uso de las TIC, hasta experiencias de práctica y desarrollo profesional, situación que con la pandemia COVID-19 ha aumentado y ha puesto a los docentes en procesos de aprendizaje profundos respecto de las nuevas tecnologías educativas que pueden compartir a sus estudiantes.

Otro elemento importante dentro de la incorporación de las TIC a los espacios educativos es comprender que los profundos cambios que se están generando referidos a las tecnologías en nuestras vidas también afectan a los procesos de formación. Esto significa la posibilidad de crear entornos virtuales de aprendizaje (EVA), bajo enfoques metodológicos no tradicionales, transitando desde un aprendizaje individual a un aprendizaje colaborativo, desde la transmisión a la construcción de conocimiento. Estos espacios se están haciendo realidad y se están convirtiendo en el nuevo escenario donde el docente debe interactuar. 
Coincidimos con Quiroz (2012) en que: "Los profesores pueden utilizar estos espacios virtuales como apoyo o complemento de una clase presencial o para desarrollar una experiencia formativa íntegramente en formato virtual”.

Cabe destacar una lectura añadida a las anteriores: la de la enseñanza-educación en valores (adoctrinamiento) merced a la propaganda, pues ésta también ha hallado cabida dentro del vehículo comunicativo llamado TIC. Trabajos como el de Gómez-López (2019) nos muestran este tipo de enseñanza aplicada a la información sobre los conflictos a nivel mundial.

\section{Discusión}

\subsection{El currículum, un cruce de prácticas diversas}

Cuando hablamos de currículum podemos decir que estamos frente a un contexto complejo de realidad social, pues existen diversas perspectivas y teorías del currículum que ponen sus énfasis en distintas dimensiones del quehacer educativo, y que de alguna u otra forma van impactando en la realidad del aula y en los aprendizajes de los estudiantes. En este sentido trataremos de aproximarnos a una teoría crítica del currículum, la cual apele a considerar el concepto de práctica educativa como una construcción social, donde la orientación crítica cuestione las propuestas técnicas que subordinan los medios a los fines, por la racionalidad misma de los fines. Además, entender que los juicios acerca de lo que deba ser la práctica educativa no sólo son internos a la propia práctica, o a lo que acuerdan los sujetos implicados, sino que precisan de instancias normativas y críticas desde las que juzgar la realidad descrita y legitimar sus líneas de transformación.

Así Carr (2002), por ejemplo, mantiene que una investigación educativa "no puede contentarse con las propias interpretaciones de los practicantes, sino que también debe estar dispuesta a evaluarlas críticamente y sugerir explicaciones alternativas que en algún sentido sean mejores". Es así como la perspectiva del currículum crítico se dirige, pues, a mostrar que el conocimiento y experiencia educativa, como práctica social, están socialmente condicionadas. Se han de desvelar dichos condicionantes para que los actores tomen conciencia y, de este modo, incidir en propuestas educativas que contribuyan a la progresiva transformación de los sujetos. De esta manera los fenómenos educativos son realidades socialmente construidas, determinadas y comprometidas con opciones de valor. Consecuentemente, las propuestas curriculares críticas no son ajenas a un contexto social y están ideológicamente comprometidas en todos los ámbitos del saber en los que existe esta necesidad, incidiendo en la oferta formativa incluso en las Universidades (Carretero-Díaz y

Perspectivas de la Comunicación - Vol. 14 - No 2 - 2021 - pp. 149-170

Universidad de la Frontera - Chile 
Barrientos-Báez, 2019). Por ello, la construcción del conocimiento se orienta a la participación y a la comunicación competente, esto quiere decir que las TIC tienen un uso educativo que se orienta a desarrollar un conocimiento constructivo donde la actividad educativa tiene dimensiones solidarias, emocionales y democráticas, involucrando así a los estudiantes en su construcción y posterior utilización.

Parece necesario prestar atención a las condiciones que faciliten o impidan la competencia comunicativa de los sujetos implicados. Así el currículum debe situarse en un espacio social amplio a través de un conjunto de mediaciones personales, institucionales y sociales. $\mathrm{Al}$ ser una práctica construida socialmente, su análisis no puede limitarse a los documentos o prácticas docentes; es preciso adentrarse en sus condicionamientos sociales y políticos. Por eso es importante enfatizar que las TIC se instalan como una herramienta en los procesos de enseñanza - aprendizaje, pero lo que está detrás de ellas es el papel del currículum situado en el contexto y espacio social. Así se puede afirmar que los cambios que se están dando a nivel estructural de la sociedad, los cambios que se den en las instituciones universitarias y en el campo educativo en general, forman parte de un proceso donde "los cambios en los estilos docentes son cambios evolutivos, paulatinos y no son producto de grandes y bruscas mutaciones (Sacristán, 1991).

\subsection{El concepto de práctica educativa}

Continuamente en el ámbito educativo se habla de práctica. No obstante, hay diversas concepciones para entenderlo. Por una parte, se puede interpretar erróneamente la noción de práctica como una mera realización de acciones consecutivas enmarcadas en un tiempo y un espacio determinado, separando así la acción de la subjetividad de quien realiza esta acción. Esta separación ha provocado la modificación del concepto de práctica. Con la pérdida del sujeto se atomiza la noción de práctica y no se le da el lugar e importancia que merece. Es así como en este artículo se ha reformulado lo que significa el concepto de práctica, y resignificado lo planteado por Wilfred Carr, quien señala que:

\footnotetext{
"Un modo de comenzar es [...] pensar en la práctica como algo construido. Aunque quizá estemos acostumbrados a pensar en la práctica como en una mera "actividad", puede demostrarse que el sentido y la significación de la práctica educativa se construye en los planos social, histórico y político, y que sólo puede entenderse de forma interpretativa y crítica. Desde este punto de vista, la práctica no es un mero "hacer". No se trata de una especie de acción técnica, instrumental; tiene unos sentidos y unas significaciones que no pueden comprenderse sólo mediante la observación de nuestras acciones. Pero su sentido y significación no son exclusivamente subjetivos (cuestión de las perspectivas y formas
} 
de comprender de los profesionales), sino que pueden ser comprendidos por los otros, interpretándolos, y se enmarcan en la historia y en la tradición, así como en la ideología”. (Carr, 2002, p. 23)

Esta explicación que muestra Carr (2002) sobre el concepto de práctica es decisoria, porque instantáneamente muestra al sujeto que se ha olvidado y se puede percatar que la práctica no puede estar disociada o desvinculada de la subjetividad de quien la experimenta, pero además es posible ser comprendida e interpretada por un tercero. De ahí que la típica frase que siempre escuchamos: "la experiencia es la madre de todas las ciencias", se debe a que es en la experiencia cotidiana donde el sujeto construye el sentido y significado a su experiencia de vivir.

Se supera así la visión dualista de sujeto - objeto, donde se ha negado la unidad dialéctica de la práctica con la teoría. Por eso, la necesidad de volver a repensar el concepto de práctica educativa desde una perspectiva dialéctica en constante movimiento. Es importante ya que se está introduciendo al sujeto en el análisis de sus producciones. La historia, la práctica y la teoría son producciones humanas y no pueden analizarse independiente del momento histórico. Esto queda explicado más claramente por Freire cuando recuerda a Marx diciendo:

"La historia no hace nada, no posee ninguna inmensa riqueza, no libra ninguna clase de lucha: quien hace todo esto, quien posee y lucha es el hombre mismo, el hombre real, vivo; no es la historia la que utiliza al hombre para alcanzar sus fines - como si se tratase de una persona aparte - porque la historia no es sino la actividad del hombre que persigue sus objetivos”. (Freire, 2008, pp. 27-28)

El recuerdo de las palabras de Marx que releva Freire muestra que los seres humanos no sobrepasaremos la condición problemática en la que estamos, por medio de nuestra conciencia o de nuestras buenas intenciones, sino que lo lograremos mediante la praxis como seres que podemos transformar el mundo. Incluso esta misma transformación es una invención humana que, si se deja fuera de los análisis de la producción del conocimiento que ha creado, no reviste de sentido. Por eso, "la praxis no es la acción ciega, desprovista de intención o de finalidad. Es acción y reflexión” (Freire, 2008, p. 28). Tanto mujeres como hombres se han hecho históricamente seres de praxis, volviéndose capaces de transformar el mundo y de conferirle significado. Así, podremos asumir una situación concreta que nos desafía a modificar el significado de nuestra realidad mediante la acción. Freire (2008) señala que: "separada de la práctica la teoría es puro verbalismo inoperante; desvinculada 
de la teoría, la práctica es activismo ciego. Es por esto mismo que no hay praxis auténtica fuera de la unidad dialéctica acción - reflexión, práctica - teoría” (p. 30).

Con todo esto, la ciencia clásica tradicional, "ha hecho reinar cada vez más a los métodos de verificación empírica y lógica. Mitos y tinieblas parecen ser rechazados a los bajos fondos del espíritu por las luces de la razón” (Morin, 1994, p. 14). Lo que dice el autor muestra que la cultura occidental ha vivido bajo el imperio de la razón donde los principios de reducción y disyunción han sido considerados como principios de verdad; es decir, se ha considerado a la objetividad de la realidad como algo cuantificable, como algo estático. Si bien esta situación ha permitido grandes avances del conocimiento científico, también ha traído consecuencias nocivas para nuestra existencia, como por ejemplo cuando se habla sobre la inteligencia ciega:

\footnotetext{
"Así es que llegamos a la inteligencia ciega. La inteligencia ciega destruye los conjuntos y las totalidades, aísla todos sus objetos de sus ambientes. No puede concebir el lazo inseparable entre el observador y la cosa observada. Las realidades clave son desintegradas. Pasan entre los hiatos que separan a las disciplinas. Las disciplinas (las ciencias humanas no necesitan más de la noción de hombre. Y los ciegos pedantes concluyen que la existencia del hombre es sólo ilusoria. Mientras los medios producen la cretinización vulgar, la Universidad produce la cretinización de alto nivel. La metodología dominante produce oscurantismo porque no hay más asociación entre los elementos disjuntos del saber y, por lo tanto, tampoco posibilidad de engranarlos y de reflexionar sobre ellos". (Morin, 1994, p. 16)
}

Según lo citado, se puede comprender que desafortunadamente la incapacidad de incorporar la complejidad al pensamiento humano ha hecho que se siga concibiendo la realidad como una cosa que está fuera de quien la percibe y la vivencia. Se da el esfuerzo por comprender la verdad de la realidad, "al tratar de conocer la realidad, el investigador crítico y vigilante no puede pretender domesticarla a sus objetivos. Lo que quiere es la verdad de la realidad, y no la sumisión de ésta a su verdad” (Freire, 2008, p. 34). Por eso, se señala que:

\footnotetext{
"la actitud vigilante que caracteriza a todo investigador crítico, el que no se satisface con apariencias engañosas. Él sabe muy bien que el conocimiento no es algo dado y acabado, sino un proceso social que exige la acción transformadora de los seres humanos sobre el mundo”. (Freire, 2008, p. 35)
}

En definitiva, "La práctica es una forma de "acción inmaterial" precisamente porque su fin sólo puede realizarse a través de la acción y sólo puede existir en la acción misma" (Carr, 2002, p. 96). Así, la práctica educativa es una relación multidimensional donde se 
involucran elementos como: dimensiones didácticas, psicopedagógicas, políticas y éticas. Ello determinado por un contexto espacio temporal que las va configurando. La práctica educativa según Lacourse (2009) es: “una praxis que integra dialécticamente la anticipación, la práctica y la reflexión crítica. Esta incluye el conjunto de acciones de planificación, seguidas de la operacionalización en la clase y de la evaluación de esta operacionalización” (p. 169).

Ahora bien, si consideramos la práctica educativa como una construcción social, propia de la vida en sociedad, cabe preguntarse ¿cómo las TIC pueden constituirse en buenas prácticas para la enseñanza - aprendizaje? ¿De qué manera pueden ayudar a que este nuevo orden globalizado no deje vacío el concepto de práctica educativa de su contenido, enfatizando sólo en lo técnico: el hacer, el fabricar, ¿el producir?

De una educación tradicional centrada en la virtud, se puede pasar a una educación entendida como la aplicación de teorías que determinan verdaderamente lo que hay que hacer para la producción de las condiciones que conduzcan a la conducta deseada.

Este mismo principio subyace como base de la estandarización del desempeño docente. De este modo, las medidas que los sistemas educativos implementen relacionadas con articular teoría y práctica no van a producir las transformaciones deseadas, mientras lo que se siga implementando sea medidas que fortalezcan las relaciones entre teoría y técnica. En tal sentido, habría que preguntar ¿qué se está entendiendo en las políticas públicas, centros de formación docente por teoría, por práctica y por sus relaciones? Además, đqué implicancias tiene en las propuestas para su articulación y estandarizar el desempeño docente?

\subsection{La didáctica no parametral: Un Nuevo Paradigma Educativo}

Si vamos comprendiendo que las prácticas educativas son acciones inmateriales y que las TIC pueden ser herramientas que facilitan los procesos de enseñanza - aprendizaje en el aula. Es necesario que este aprendizaje apoyado por las TIC considere la dimensión didáctica con lo que se construye el conocimiento. Es en este horizonte que se comenzará a introducir una temática no muy abordada en nuestro país, y es la que refiere a la Didáctica No Parametral como un nuevo paradigma educativo. Este nuevo paradigma proviene del trabajo realizado por la maestra argentina Estela Quintar, la que ha propuesto una nueva manera de diseñar escenarios propicios para la construcción del conocimiento. A grandes rasgos la didáctica no parametral propone un proceso de enseñanza de una manera menos

Perspectivas de la Comunicación - Vol. 14 - No 2 - 2021 - pp. 149-170

Universidad de la Frontera - Chile 
esquemática, rígida y con un estado anímico en positivo, en conciencia histórica, en el cual se reclama a un sujeto erguido y en postura que exige unas visiones sobre la relación con el conocimiento distinta. Es así como se señala lo siguiente:

\footnotetext{
"La didáctica no-parametral es una postura que comprende el proceso de enseñanza como un proceso intencional de permanente promoción de ruptura de sentidos y significados en el propio devenir existencial; lo que implica definir: Al conocimiento como construcción de sentidos y significados, es decir, de redes de representaciones simbólicas históricas e historizadas. Al sujeto concreto como sujeto atado a su territorialidad contextual y a su subjetividad, lo que hace de él un sujeto dialéctico en tanto que se articula en su sujeción simbólica, como sujeto de deseo y como sujeto del inconsciente, sujeciones que se actúan en el mundo de relaciones situadas, en este caso, en situaciones de enseñanza aprendizaje concretas”. (Quintar, 2002, p.12)
}

Tal como lo señala la autora, la didáctica no parametral es una propuesta alternativa al paradigma educativo tradicional, el cual apela a cuestionarse el cómo construimos el conocimiento y para qué aprendemos. En este sentido y si nuestro propósito recae en el análisis didáctico de los procesos de enseñanza - aprendizaje que utilizan las TIC como herramientas educativas, no podemos perder los referentes epistemológicos que nos orientan hacia cómo debe realizarse el proceso educativo. La incorporación de las TIC como mediador del proceso de aprendizaje nos lleva a valorar y a reflexionar sobre la eficacia de la enseñanza. La didáctica se ha constituido como el ámbito de organización de las reglas de método para hacer que la enseñanza sea eficaz. Quizá, esa sea una de las razones de asimilación con el "cómo" de la enseñanza, cuestión que ha llevado al solapamiento de ésta con la cuestión metodológica, un aspecto de la didáctica que ha sido escasamente abordado en los últimos años.

\subsection{La Didáctica entre las Ciencias de la Educación}

Es necesario, entonces, determinar y valorar algunos aspectos relevantes acerca del concepto de Didáctica. La didáctica actual sigue la línea del conocimiento basada en la investigación de preguntas teóricas y prácticas, que se centran sobre todo en los procesos de enseñanza y aprendizaje, donde se establecen cuatro ejes de construcción de la didáctica:

- El marco epistemológico o pedagógico como espacio disciplinar e interdisciplinar con características propias.

- El marco contextual, con un espacio operativo próximo a la comunidad educativa o espacio operativo ampliado. 
- El marco curricular, con la intención profesionalizadora del marco curricular en el que cabe el profesor.

- El marco pragmático, en donde el estudiante es el beneficiario directo y más significativo de la acción didáctica.

Es precisamente este contexto, el pedagógico, el universitario, del profesor y de los estudiantes, en el que actúa la didáctica y el que se erige en contexto de legitimación epistemológica y a la vez, como contexto de significación. De este modo, se considera la didáctica como una ciencia o campo científico que tiene por objeto de estudio la mejora de los procesos de enseñanza - aprendizaje, con la finalidad de contribuir al desarrollo personal del estudiante. Teniendo en cuenta las anteriores ideas sobre didáctica, la propuesta pedagógica se basa fundamentalmente en la didáctica no parametral como propone Quintar (2002), quien plantea una ruptura de esquemas y de parámetros de la educación tradicional; fragmentar los esquemas y visualizar un contexto real, brindando una educación integral que parta de la subjetividad del estudiante y, por tanto, lo lleve a su desarrollo pleno en la sociedad actual. Para iniciar este viaje es necesario reconocer el objetivo educativo, el cual es desarrollar el pensamiento de los sujetos, enseñar a pensar y que el aprendizaje se desarrolle en el nivel del conocer, hacer y ser del sujeto. Es aquí donde es necesario retomar lo dicho por Freire (2002), en su libro Pedagogía de la autonomía, que propone al docente como un maestro y un aprendiz permanente, en una relación disciplinada, pero libre a la vez, donde el estudiante tiene la función de ser y el maestro de aceptar la diversidad, además de desarrollar su capacidad investigativa. En este contexto las TIC son propicias para que los procesos de enseñanza - aprendizaje construyan conocimiento autónomo.

Es así como la didáctica no parametral está constituida por componentes, entendidos por contenidos didácticos. El contenido didáctico debe entenderse como todo aquello posible de ser enseñado y aprendido. La selección de contenidos de enseñanza implica y se compromete con el conocimiento, con un ángulo epistémico de razonamiento en la construcción de lo real, del mundo, de la vida misma de una sociedad.

Esta propuesta pedagógica considera la dimensión histórica que se está dando, en un presente coyuntural y potencial. 


\section{Conclusiones}

La didáctica no parametral es una estrategia que resulta apropiada para los contextos universitarios, más aún con la intervención de las TIC, pues lleva a adquirir habilidades metacognitivas. Con este modelo de didáctica se brinda la oportunidad de explorar acciones dentro y fuera del aula, tanto de los estudiantes como del docente, cambiando rutinas, modelos y caminos de aprendizaje, permitiendo que los sujetos implicados en el proceso asuman nuevos compromisos y retos.

Dado que las instituciones educativas se caracterizan por argumentar la falta de material didáctico, es indispensable que los docentes estén capacitados para desarrollar acciones alternativas, contando con la interacción permanente de la realidad inmediata del sujeto, aprovechando el gran laboratorio que ofrece la naturaleza e incluyendo los eventos diarios y las experiencias de los estudiantes.

Es pertinente continuar desarrollando procesos metacognitivos aplicados a una didáctica no parametral aprovechando el entorno como escenario de aprendizaje; no obstante, se ha de tener en cuenta el ajuste a los instrumentos de apoyo en los cuales se considere el control externo como factor de influencia en la adquisición de autonomía de los estudiantes.

\section{Conflicto de interés}

Los autores declaran que no existe conflicto de interés. 


\section{Referencias bibliográficas}

BARRIENTOS-BÁEZ, A. (2016). GDS Amadeus. Propuesta de innovación didáctica. En J. F. Durán y I. Durán (directores.), TIC actualizadas para una nueva docencia universitaria (pp. 17-30). McGraw Hill.

BARRIENTOS-BÁEZ, A., Barquero-Cabrero, M. \& Rodríguez-Terceño, J. (2019). La educación emocional como contenido transversal para una nueva política educativa: el caso del grado de turismo. Revista Utopía y Praxis Latinoamericana, 24(4), pp. 147-165.

https://produccioncientificaluz.org/index.php/utopia/article/view/29796

BARRIENTOS-BÁEZ, A., CALDEVILLA-DOMÍNGUEZ, D. \& GARCÍA, E. (2017). APP para la tercera edad: utilidad, clases y valor social. Revista de Ciencias de la Comunicación e Información, 22(2), pp. 1-11.

http://doi.org/10.35742/rcci.2017.22(2).1-11

BLANCO, A. (2018). Comunicación, Redes de conocimiento y cooperación: Revisión teórica y propuesta metodológica. Perspectivas de la Comunicación, 11(2), pp. 231-250.

http://revistas.ufro.cl/ojs/index.php/perspectivas/article/view/1920/1751

BOTELLA-NICOLÁS, A. M., HURTADO-SOLER, A. \& RAMOS-AHIJADO, S. (2019). Innovación y TIC en el paisaje sonoro de la música festera a través de la creación de musicomovigramas. Vivat Academia, Revista de Comunicación, 147, pp. 109123. http://doi.org/10.15178/va.2019.147.109-123

CALDEVILla-DOMÍNGUEZ, D., BARRIENTOS-BÁEZ, A. \& PARRA-LÓPEZ, E. (2020). Horizontes del mundo digital: de la simulación y la banalización de la experiencia, un uso social, ecológico e innovador de la Sociedad Red. CIC. Cuadernos de Información y Comunicación, 25, pp. 269-277. http://dx.doi.org/10.5209/ciyc.68722

CALDEVILLA-DOMÍNGUEZ, D., RODRÍGUEZ-TERCEÑO, J. \& BARRIENTOS-BÁEZ, A. (2019). El malestar social a través de las nuevas tecnologías: Twitter como 
herramienta política. Revista Latina de Comunicación Social, 74, pp. 1264-1290. http://doi.org/10.4185/RLCS-2019-1383-66

CARR, W. (2002). Una Teoría para La Educación. Hacia una Investigación Educativa Crítica. Ediciones Morata.

CARRETERO-DÍAZ, M. A. \& BARRIENTOS-BÁEZ, A. (2019). Necesidad de formar a los cuidadores de personas en situación de dependencia: comunicación y respuesta desde la UCM. Revista de Comunicación y Salud, 9(1), pp. 19-38. http://doi.org/10.35669/revistadecomunicacionysalud.2019.9(1).19-38

CASANOVA-CORREA, J. (2007). Desafíos a la formación inicial del profesorado: buenas prácticas educativas en el contexto de la innovación con TIC. Revista Latinoamericana de Tecnología Educativa, 6(2), pp. 109-125.

CELAYA, I., RAMÍREZ-MONTOYA, M. S., NAVAL, C. \& ARBUÉS, E. (2020). Usos del podcast para fines educativos. Mapeo sistemático de la literatura en WoS y Scopus (2014-2019). Revista Latina de Comunicación Social, 77, pp. 179-201. www.doi.org/http://doi.org/10.4185/RLCS-2020-1454

COLÁS-BRAVO, P., ROSSI, P. G., PABLOS-PONS, J., CONDE-JIMÉNEZ, J. \& VILLACIERVOS-MORENO, P. (2019). Aplicaciones digitales para la inclusión. El Proyecto Europeo DEPIT. Revista de Comunicación de la SEECI, 50, pp. 169192. http://doi.org/10.15198/seeci.2019.50.169-192

FANDOS-GARRIDO, M., JIMÉNEZ, J. \& GONZÁLEZ-SOTO, A. (2002). Estrategias didácticas en el uso de las tecnologías de la información y la comunicación. Revista Acción Pedagógica, 11(1), pp. 28-39.

https://dialnet.unirioja.es/servlet/articulo?codigo $=2973066$

FERNÁNDEZ-RUIZ, B. (2020). Innovación educativa mediante la gestión emocional. Revista de Ciencias de la Comunicación e Información, 25(3), 41-56. http://doi.org/10.35742/rcci.2020.25(3).41-56

FREIRE, P. (2002). Pedagogía de la Autonomía. Saberes necesarios para la práctica 
educativa. Siglo XXI.

(2008). La Importancia de Leer y el Proceso de Liberación. Siglo XXI

GALLEGO-TORRES, R. A. (2020). Diseño, producción e implementación del ambiente de aprendizaje basado en web, AABW. Revista de Comunicación de la SEECI, 52, pp. 119-147. http://doi.org/10.15198/seeci.2020.52.119-147

GARCÍA-MARÍN, D. (2020). Universo transpodcast. Modelos narrativos y comunidad independiente. Historia y Comunicación Social, 25(1), pp. 139-150. https://doi.org/10.5209/hics.69232

GIL-VILLA, F., URCHAGA, J. D. \& SÁNCHEZ-FERNÁNDEZ, A. (2020). Proceso de digitalización y adaptación a la enseñanza no presencial motivada por la pandemia de COVID-19: análisis de la percepción y repercusiones en la comunidad universitaria. Revista Latina de Comunicación Social, 78, pp. 99119. www.doi.org/10.4185/RLCS-2020-1470

GÓMEZ-LÓPEZ, J. (2019). Tecnologías de la información y los mensajes en los nuevos espectros del conflicto. Revista de Ciencias de la Comunicación e Información, 24(2), pp. 45-56. http://doi.org/10.35742/rcci.2019.24(2).45-56

HERNÁNDEZ-QUINTANA, A. R. (2019). Espacios críticos de responsabilidad social para las Humanidades Digitales. Bibliotecas. Anales de Investigación, 15(1), pp. 121-125. https://bit.ly/3j89wzd

LACOURSE, F. (2009). Rutinas profesionales en la Educación Primaria y Secundaria: Contribución a las competencias de las prácticas de enseñanza. Revista Pensamiento Educativo, 44(45), pp. 159-183.

MANIGLIO, F. (2016). El ataque tecnocrático: las políticas de comunicación y educación en la Unión Europea. Perspectivas de la Comunicación, 9(1), pp. 742.

http://publicacionescienciassociales.ufro.cl/index.php/perspectivas/article/vie w/634 
MASCARELL-PALÁU, D. (2019). Implementación y uso de las TIC. Dispositivos móviles en educación en artes. Un estado de la cuestión. Revista de Comunicación de la SEECI, 50, pp. 73-86.

http://doi.org/10.15198/seeci.2019.50.73-86

(2020). Fomento del Mobile Learning en educación alrededor de la última década. Un estudio de caso en España través de una selección de aportaciones. Vivat Academia. Revista de Comunicación, 153, pp. 73-97.

https://doi.org/10.15178/va.2020.153.73-97

MEARS-DELGADO, B. \& MARZAL, M. Á. (2018). Evalfin: un modelo de evaluación de alfabetización informacional para instituciones de educación superior. El Profesional de la Información, 27(4), pp. 879-890.

https://doi.org/10.3145/epi.2018.jul.16

MONZALVO, A., ROJAS, D. \& TORRES, N. Y. (2018). Validación de la escala de percepción de inclusión docente de la niñez migrante (PID). Revista de Comunicación y Salud, 8(1), pp. 61-70.

http://doi.org/10.35669/revistadecomunicacionysalud.2018.8(1).61-70

MOREIRA, J. A., LIMA-SANTANA, C. \& GONZÁLEZ-BENGOECHEA, A. (2019). Ensinar e aprender nas redes sociais digitais: o caso da MathGurl no YouTube. Revista de Comunicación de la SEECI, 50, pp. 107-127. http://doi.org/10.15198/seeci.2019.50.107-127

MORIN, E. (1994). Introducción al pensamiento complejo. Gedisa.

NOVOA, P. F. (2019). Estrategias de aplicación digital en la comprensión de textos narrativos. Investigaciones sobre Lectura, 11, pp. 37-55. http://doi.org/10.37132/isl.voi11.277

ORTIZ, M. Á., MARTA, C. \& GABELAS, J. A. (2019). Los niños y adolescentes ante las pantallas: el cambio de paradigma en el modelo de relaciones y mediación. Historia y Comunicación Social, 24(1), pp. 353-365.

https://doi.org/10.5209/hics.64499 
PONTES, N., DORADO-SANTANA, Y. \& BAPTISTA-CALUNGA, A. (2020). A gestão documental em instituições escolares: directrizes para o desenho de um sistema de gestão electrónica de documentos escolares. Bibliotecas. Anales de Investigación, 16(1), pp. 23-45. www.bnjm.cu/revista-anales/index.php

QUINTAR, E. (2002). La Enseñanza como puente a la vida. Universidad Nacional de Pachuca.

QUIROZ, J. S. (2012). Estándares TIC para la Formación inicial Docente: Una política pública en el contexto chileno. Education Policy Analysis Archives/Archivos Analíticos de Políticas Educativas, 20, pp. 1-36. https://doi.org/10.14507/epaa.v20n7.2012

RAJAS, M., PUEBLA-MARTÍNEZ, B. \& BAÑOS, M. (2018). Formatos audiovisuales emergentes para MOOC: diseño informativo, educativo y publicitario. El Profesional de la Información, 27(2), pp. 312-321. https://doi.org/10.3145/epi.2018.mar.o9

SACRISTÁN, G. (1991). El Currículum: Una reflexión sobre la práctica. Ediciones Morata.

SALAS-RUEDA, R. A. \& SALAS-RUEDA, R. D. (2019). Análisis sobre el uso de la red social Facebook en el proceso de enseñanza-aprendizaje por medio de la ciencia de datos. Revista de Comunicación de la SEECI, 50, pp. 1-26. http://doi.org/10.15198/seeci.2019.50.1-26

SALAS-RUEDA, R. A. \& SALAS-SILIS, J. A. (2019). WeVideo: ¿Servicio en la nube útil para los estudiantes durante la construcción y presentación de los contenidos audiovisuales? Vivat Academia. Revista de Comunicación, 149, pp. 67-89. http://doi.org/10.15178/va.2019.149.67-89

SALAS-RUEDA, R. A. \& VÁZQUEZ-ESTUPIÑÁN, J. J. (2017). Aplicación en la nube Lucidchart: ¿herramienta necesaria para la innovación del proceso educativo en el siglo XXI? Revista de Comunicación de la SEECI, 44, pp. 115-126. 


\section{http://dx.doi.org/10.15198/seeci.2017.44.115-126}

TABERNERO, R., ÁLVAREZ, E. \& HEREDIA, H. (2020). Hábitos de lectura y consumo de información de los adolescentes en el ámbito digital. Investigaciones sobre Lectura, 13, pp. 72-89. http://doi.org/10.37132/isl.voi13.302

TAVARES, D. (2019). Diretrizes para uma política de comunicação científica para a Universidade do Estado de Mato Grosso (Brasil). Perspectivas de la Comunicación, 12(1), pp. 135-156. https://doi.org/10.4067/So71848672019000100135

VALBUENA-BOHÓRQUEZ, A. R. \& ALVARADO-ORTIZ, J. J. (2020). La interactividad de las herramientas tecnológicas en el desarrollo del pensamiento lógico en educación básica secundaria. Revista de Ciencias de la Comunicación e Información, 25(3), pp. 1-17. http://doi.org/10.35742/rcci.2020.25(3).1-17

VIDAL-SANHUEZA, J. A., PONCE, M. P., CIFUENTES, K. D. \& VIÑUELA-ALLER, R. (2009). Usos, integración curricular y adopción tecnológica de la informática educativa en las prácticas pedagógicas de docentes de La Araucanía, Chile. Revista Iberoamericana de Educación, 49(5), pp. 1-12. https://doi.org/10.35362/rie4952065 